\title{
Information Consensus of Asynchronous Discrete-time Multi-agent Systems
}

\author{
Lei Fang and Panos J. Antsaklis
}

\begin{abstract}
This paper studies the consensus problem of multi-agent systems in an asynchronous framework. Under certain assumptions, the consensus protocol leads to stable behaviors even if the updating instants and sets of the agents are asynchronously determined. The model of asynchronous multi-agent systems encompasses those synchronous ones with various communication patterns, i.e., issues of directional, delayed, or failed communication can be addressed in the same framework. The asynchronous results in this paper thus shed new light on the synchronous results reported in the literature. In particular, synchronous protocols under dynamically changing interaction topologies can be seen as a special case of the asynchronous protocol where all communication delays are zero.
\end{abstract}

\section{INTRODUCTION}

Consensus is well accepted as being a fundamental paradigm for coordination of groups of autonomous agents [3], [14], [19], [26], [28], [31]. In such a system agents are autonomous enough to operate independently, yet they can function collectively as a group and synchronize through communication. A consensus protocol provides means through which all agents are coordinated in the sense that they all agree on some particular parameter of interest. Our interest in multi-agent systems is motivated by their broad applications in formation control [7], [9], collective motion [40], cooperative robotics [1], distributed space systems [38], and sensor networks [34]. The challenge here is for the group to cooperate using minimal computing and communication resources, without the need for centralized coordination. Naturally, the information flow and interaction among multiple agents in the group play an important role in the coordinated behavior of these agents.

In the past, a number of researchers have worked in problems that are closely related to consensus problems. Distributed agreement problems in Computer Science have a long history [24]. Coordinated behaviors in nature, such as flocking/swarming and coupled oscillators, have been studied in ecology and biology, as well as in statistical physics and nonlinear science [29], [40]. Engineering applications such as formation control have further increased the interest of engineers in swarming behaviors and collective motion patterns [9], [11], [14], [16], [19]. Very recent results on consensus problems include [2], [3], [6], [12], [14], [17], [19], [25]-[28], [31], [33], [35], [41], to name a few. These results can be broadly divided into several categories according to the main mathematical tools used: algebraic graph theory (e.g., [14], [28], [31]), nonlinear dynamics

L. Fang and P. J. Antsaklis are both with Dept. of Electrical Engineering, Univ. of Notre Dame, Notre Dame, IN 46556. E-mail: $\{$ lfang, antsaklis. 1 \}and.edu. This work was supported by the Center for Applied Mathematics, Univ. of Notre Dame.
[26], [27], convex or iterative optimization [6], [25], [41], and partial contraction theory [35].

In the following, we briefly review the work most relevant to the present research and then point out the relationships among them. The work in [14] focuses on attitude alignment on undirected graphs in which the agents have simple dynamics motivated by the model used in [40]. It is shown that the consensus on the heading angles of the agents can be achieved if the union of the interaction graphs for the team are connected frequently enough as the system evolves. Average-consensus problem is solved in [28] over directed graphs, which are required to be strongly connected and balanced. Ren et al. [31] extend the results of [14] from the bidirectional case to unidirectional case. If the union of the collection of interaction graphs across some time interval had a spanning tree frequently enough, consensus (not necessarily average-consensus) can still be achieved. The interesting work of [26], [27] follows a rather different approach from the previous ones, where tools from nonlinear dynamics are used to obtain a broad class of communication patterns that guarantee global consensus. A distributed iterative procedure under virtual synchronization (a weak form of asynchronism) is developed by Mehyar et. al for calculating averages over an unstructured peer-topeer network [25]. Other complementary research efforts are being reported in [17], [19], [33].

The consensus protocols aforementioned cannot be regarded as truly distributed because each agent's decisions must be synchronized to a common clock shared by all other agents in the group. This synchronization requirement could be unrealistic. Motivated by this, we study the consensus problem for asynchronous discrete-time multi-agent systems. In such systems each agent operates according to its own clock; no assumption is made about the relative speeds of different clocks. Agents communicate solely by sending messages, however there is no guarantee on the time of delivery or even of a successful delivery. This scenario is actually prevalent in real systems such as distributed networks. We refer readers to [5], [10], [15] for surveys on the theory of asynchronous systems.

The asynchronism can destroy convergence properties that the algorithm may possess when executed synchronously or sequentially. Thus, the analysis of asynchronous algorithms is considerably more difficult than of their synchronous counterparts. Nevertheless, asynchronous systems give more reasonable models of the multi-agent systems in practical situations. For instance, heterogeneous agents, time-varying communication delays and packet dropout can be taken into account without much difficulty using the results from asynchronous theory. 
In this work, we propose an asynchronous framework to study the consensus problem. To facilitate the analysis, we start with the multi-agent systems under fixed interaction topologies, which have a rooted directed spanning tree. The spanning tree requirement is a milder condition than connectedness and is therefore more suitable for practical applications [7], [31]. Under certain assumptions, even if the updating instants of the agents are asynchronously determined, the consensus protocol continues to maintain the stability property. Despite its simple fixed topology, the model of asynchronous multi-agent systems encompasses those synchronous ones with various communication patterns, i.e., we can address issues of directional, delayed, or time-variant communication in the same framework.

This work is not the first one that uses the asynchronous models to analyze multi-agent systems; see, e.g., [4], [18], [20]-[22]. Another related work is presented in [39], where decentralized asynchronous control and optimization schemes for stochastic discrete-event systems are analyzed. In the present paper, synergistic union of graph theory, matrix theory and asynchronous theory allows us to reach very general results on the consensus problem.

The contributions of this paper are two-fold. First, we extend the existing (synchronous) consensus results to the asynchronous setting. Under certain mild conditions, consensus can still be achieved for asynchronous multi-agent systems. Our asynchronous results shed new light on the existing (synchronous) results reported in [14], [25], [26], [28], [31]. Second, connections between synchronous and asynchronous protocols are established. As we show in this paper, the asynchronous protocol under fixed topologies contain synchronous protocols under time-varying topologies as a special case. Then many of the techniques and results obtained earlier for asynchronous systems can be applied to study synchronous protocols, thus providing us with deeper understanding of the synchronous consensus dynamics. For example, the robustness properties of the synchronous protocol under time-varying topologies can be examined using the asynchronous theory.

\section{PRELIMINARIES AND BACKGROUND}

\section{A. Definitions and Notations}

Let $G=\{V, E, A\}$ be a weighted digraph (or direct graph) of order $n$ with the set of nodes $V=\left\{v_{1}, v_{2}, \ldots, v_{n}\right\}$, set of edges $E \subseteq V \times V$, and a weighted adjacency matrix $A=\left[a_{i j}\right]$ with nonnegative adjacency elements $a_{i j}$. The node indices belong to a finite index set $\mathscr{I}=\{1,2, \ldots, n\}$. A directed edge of $G$ is denoted by $e_{i j}=\left(v_{i}, v_{j}\right)$. For a digraph, $e_{i j} \in E$ does not imply $e_{j i} \in E$. The adjacency elements associated with the edges of the graph are positive, i.e., $a_{i j}>0$ if and only if $e_{j i} \in E$. Moreover, we assume $a_{i i} \neq 0$ for all $i \in \mathscr{I}$. The set of neighbors of node $v_{i}$ is the set of all nodes which point (communicate) to $v_{i}$, denoted by $N_{i}=$ $\left\{v_{j} \in V:\left(v_{j}, v_{i}\right) \in E\right\}$.

A digraph $G$ can be used to model the interaction topology among a group of agents, where every graph node corresponds to an agent and a directed edge $e_{i j}$ represents a unidirectional information exchange link from $v_{i}$ to $v_{j}$, that is, agent $j$ can receive information from agent $i$. The interaction graph represents the communication pattern at certain time. The interaction graph is time-dependent since the information flow among agents may be dynamically changing. Let $\bar{G}=\left\{G_{1}, G_{2}, \ldots, G_{M}\right\}$ denote the set of all possible interaction graphs defined for a group of agents. Note that the cardinality of $\bar{G}$ is finite. The union of a collection of graphs $\left\{G_{i_{1}}, G_{i_{2}}, \ldots, G_{i_{m}}\right\}$, each with vertex set $V$, is a graph $\mathbb{G}$ with vertex set $V$ and edge set equal to the union of the edge sets of $G_{i_{j}}, j=1, \ldots, m$.

A directed path in graph $G$ is a sequence of edges $e_{i_{1} i_{2}}$, $e_{i_{2} i_{3}}, e_{i_{3} i_{4}}, \cdots$ in that graph. Graph $G$ is called strongly connected if there is a directed path from $v_{i}$ to $v_{j}$ and $v_{j}$ to $v_{i}$ between any pair of distinct vertices $v_{i}$ and $v_{j}$. Vertex $v_{i}$ is said to be linked to vertex $v_{j}$ across a time interval if there exists a directed path from $v_{i}$ to $v_{j}$ in the union of interaction graphs in that interval. A directed tree is a directed graph where every node except the root has exactly one parent. A spanning tree of a directed graph is a tree formed by graph edges that connect all the vertices of the graph.

Let $x_{i} \in \mathbb{R}, i \in \mathscr{I}$ represent the state associated with agent $i$. A group of agents is said to achieve global consensus asymptotically if for any $x_{i}(0), i \in \mathscr{I},\left\|x_{i}(t)-x_{j}(t)\right\| \rightarrow 0$ as $t \rightarrow \infty$ for each $(i, j) \in \mathscr{I}$. Besides being of interest in its own right, if consensus is attainable (all agents converge to a common point), then other formations are achievable too [19]. So the focus is on convergence to a point.

Let 1 denote an $n \times 1$ column vector with all entries equal to 1 . Let $M_{n}(\mathbb{R})$ represent the set of all $n \times n$ real matrices. A matrix $F \in M_{n}(\mathbb{R})$ is nonnegative, $F \geq 0$, if all its entries are nonnegative, and it is irreducible if and only if $(I+$ $|F|)^{n-1}>0$. Furthermore, if all its row sums are $+1, F$ is said to be a (row) stochastic matrix.

\section{B. Synchronous and Asynchronous Consensus Protocols}

We consider the following (synchronous) discrete-time consensus protocol [28], [31]

$$
x_{i}(t+1)=\frac{1}{\sum_{j=1}^{n} A_{i j}(t)} \sum_{j=1}^{n} A_{i j}(t) x_{j}(t)
$$

where $t \in\{0,1,2, \cdots\}$ is the discrete-time index, $(i, j) \in \mathscr{I}$ and $A_{i j}(t)>0$ if information flows from $v_{j}$ to $v_{i}$ at time $t$ and zero otherwise, $\forall j \neq i$. The magnitude of $A_{i j}(t)$ represents possibly time-varying relative confidence of agent $i$ in the information state of agent $j$ at time $t$ or the relative reliabilities of information exchange links between them. We can rewrite (1) in a compact form

$$
x(t+1)=F(t) x(t)
$$

where $x=\left[x_{1}, \cdots, x_{n}\right]^{T}, F=F_{i j}$ with $F_{i j}=\frac{A_{i j}(t)}{\sum_{j=1}^{n} A_{i j}(t)},(i, j) \in$ $\mathscr{I}$. An immediate observation is that the matrix $F$ is a nonnegative stochastic matrix, which has an eigenvalue at 1 with the corresponding eigenvalue vector equal to $\mathbf{1}$. 
The protocol (1) or (2) is localized and synchronous in the sense that all the agents update their states at the same time using the latest values of neighbors' states. From a practical point of view, since a central synchronizing clock may not exist and communication links create and fail dynamically, it is of interest to consider asynchronous multi-agent systems. In the asynchronous setting the order in which states of agents are updated is not fixed and the selection of previous values of the states used in the updates is also not fixed.

Now let $t_{0}<t_{1}<\cdots<t_{n}<\cdots$ be the time instants when the state of the multi-agent system undergoes change. Let $x_{i}(k)$ denote the state of agent $i$ at time $t_{k}$. The index $k$ is also called the event-based discrete time index in the literature. The dynamics of asynchronous systems can be written as

$x_{i}(k+1)= \begin{cases}\sum_{j=1}^{n} F_{i j}(k) x_{j}(k-d(i, j, k)) & \text { if } i \in S(k), \\ x_{i}(k) & \text { otherwise, }\end{cases}$

where $d(i, j, k) \geq 0$ are nonnegative integers, $S(k)$ are nonempty subsets of $\{1, \cdots, n\}$, the initial states are specified by $x(0)=x(-1)=\cdots$ Henceforth, we write the initial vector $x(0)$ to abbreviate reference to this set of equal initial states. We refer to the $d(i, j, k)$ as iteration delays and $S(k)$ as updating sets.

Remark 1: The delays $d(i, j, k)$ in (3) are time-varying and link-dependent. Consensus problems with time-delays have been considered by previous research, e.g., in [27], [28], but the delays considered were constant for all communication links.

\section{General Stability Theorems for Asynchronous Systems}

In this section, we focus on linear asynchronous systems satisfying the regularity assumption:

- There exists a nonnegative integer $D$ such that

$$
0 \leq d(i, j, k) \leq D<\infty, \forall(i, j, k) .
$$

Condition (4) indicates that only a finite number of updating instants can occur within any time interval of finite length. In the literature, this is also called partially asynchronism or (uniformly) bounded-delay asynchronism.

- The updating sets $S(k)$ satisfy

$$
\bigcup_{k=K}^{\infty} S(k)=\{1, \cdots, n\}, \text { for any } K .
$$

Condition (5) says that every agent is updated infinitely often. In other words, no agent fails to be updated as time goes on.

The following results due to Chazan and Miranker [8], Su et al. [36], and Lubachevsky and Mitra [23] are fundamental in the theory of stability of asynchronous systems.

Lemma 1: Let $F(k)=\left[F_{i j}(k)\right]=F, \forall k$. The fixed point of (3) is asymptotically stable under the class of regular asynchronisms if the spectral radius of $|F|, \rho(|F|)$, is less than unity. Here the absolute operation is understood to be element-wise.
Remark 2: When the context is clear, the absolute operation $|\cdot|$ applied on $F$ is dropped hereafter since it is always nonnegative in our setting.

A commonly, but mistakenly, held belief is that the condition $\rho(|F|)<1$ is also necessary for the convergence of asynchronous iterations [5], [8], [36]. The source of this error was identified only recently by Szyld in [37]. If $\rho(|F|)=1$, under certain conditions, convergence can still be achieved. Lubachevsky and Mitra [23], and more recently Pott [30], studied asynchronous methods with singular matrices, i.e., for the specific case of $\rho(F)=1$ and gave conditions for the convergence of the asynchronous iteration (3). Additional assumptions to (4) and (5) made in [23] are as follows. For at least one agent, say $i, i \in \mathscr{I}$,

$$
\begin{aligned}
F_{i i} & >0, \\
d(i, i, k) & =0, \\
x_{i}(0) & >0 .
\end{aligned}
$$

Lemma 2 ([23]): Consider the asynchronous system (3) with a nonnegative irreducible matrix $F$ of spectral radius unity. Assume (4)-(8). Then, there exists a positive, finite constant $b$ such that, as $k \rightarrow \infty, x(k) \rightarrow b \pi$, where $\pi$ is a (column) vector satisfying $F \pi=\pi$. The constant $b$ is bounded (from below and above) and its value depends on $x(0), F$, and the sequence of the update sets $S(k)$ and delays $d(i, j, k)$. Furthermore, a measure of the projective distance of $x(k)$ from the consensus point vanishes at least at a geometric rate.

\section{ASYNCHRONOUS INFORMATION CONSENSUS}

In this section, the asynchronous information consensus problem is studied. We focus on the asynchronous consensus protocol under a (structurally) fixed topology which has a rooted directed spanning tree.

\section{A. Synchronous Case with Fixed Topologies}

Let us first review the known synchronous consensus results with fixed interaction topologies. We present them here to allow for a comparison with the asynchronous model in the following sections.

Lemma 3 ([31]): Let $F \in M_{n}(\mathbb{R})$ be a stochastic matrix with positive diagonal entries. The matrix $F$ has a unique eigenvalue at 1 with maximum modulus if and only if the graph associated with $F$ has a spanning tree. In this case, $\lim _{m \rightarrow \infty} F^{m}=\mathbf{1} \mu^{T}$, where $m \in \mathbb{N}^{+}$and $\mu=\left[\mu_{1}, \ldots, \mu_{n}\right]^{T} \geq 0$ satisfies $F^{T} \mu=\mu$ and $\mathbf{1}^{T} \mu=1$.

Remark 3: The diagonal elements of $F$ are positive since we assume that there is a link from each vertex to itself.

Lemma 4 ([13], [32]): If graph $\mathrm{G}$ has a spanning tree, then

$$
\left(F-\mathbf{1} \mu^{T}\right)^{m}=F^{m}-\mathbf{1} \mu^{T}, \forall m \in \mathbb{N}^{+},
$$

where $\mu$ satisfies the properties defined in Lemma 3 . Furthermore, $\rho\left(F-\mathbf{1} \mu^{T}\right)<1$.

With the help of Lemma 3 and 4, the following synchronous stability result can be obtained. 
Theorem 1: Given the synchronous protocol (1) with $F(k)=F, \forall k \in \mathbb{N}$, the consensus is asymptotically reachable if and only if the associated interaction graph $G$ has a spanning tree. That is, global consensus is asymptotically reachable.

Theorem 1 is originally due to Ren and Beard [32]. A slightly different proof is outlined below for self-contained purposes.

Proof: From Lemma 3, we know that $F^{m} \rightarrow \mathbf{1} \mu^{T}$ as $m \rightarrow \infty$ and $F^{T} \mu=\mu, \mathbf{1}^{T} \mu=1$.

Let $\tilde{x}=x-\mathbf{1} c$, where $c=\mu^{T} x(0)$ is a real constant. We refer to $\tilde{x}$ as the (group) disagreement vector. Considering the synchronous protocol (2), we get

$$
\begin{aligned}
\tilde{x}(t) & =x(t)-\mathbf{1} c \\
& =F^{t} x(0)-\mathbf{1} \mu^{T} x(0)=\left(F-\mathbf{1} \mu^{T}\right)^{t} x(0) .
\end{aligned}
$$

It is then obvious that

$$
\tilde{x}(t+1)=\left(F-\mathbf{1} \mu^{T}\right) \tilde{x}(t), \forall t \in \mathbb{N}^{+} .
$$

From Lemma 4 , we have $\tilde{x}(k) \rightarrow 0$, or equivalently $x(k) \rightarrow$ $1 c$, as $t \rightarrow \infty$. In other words, the global consensus is asymptotically reachable in the synchronous mode.

\section{B. Asynchronous Case with Fixed Topologies}

As we shall demonstrate below, the convergence process of asynchronous protocol (3) is fundamentally different from that of the synchronous protocol (1).

A plausible condition for asynchronous consensus is $\rho\left(\left|F-\mathbf{1} \mu^{T}\right|\right)<1$, motivated by (9) and Lemma 1. However, this ignores the fact that the asynchronous system, if convergent, will not necessarily reach the same consensus point as the synchronous system does. By the necessity part of Lemma 3, to reach a consensus the matrix $F$ must have a spectral radius equal to one. Hence, we are dealing with a singular $(\rho(F)=1)$ asynchronous protocol and Lemma 2 , instead of Lemma 1, should be invoked to get a correct condition.

Before using Lemma 2, let us first check whether or not the assumptions (6)-(8) are satisfied for the asynchronous system (3). The assumption (6) is satisfied by Remark 3. In the multi-agent application, it is reasonable to assume that every agent always uses its latest state to calculate the new state, i.e., the condition $d(i, i, k)=0$ is true for at least one agent. Finally, the assumption (8) may not be a practical restriction at all if the simple expedient of taking all initial states positive is followed. It is needed to guarantee the consensus points to be bounded away from the trivial solution 0 .

Now we are ready to state the convergence result for the asynchronous protocol, which is based on Lemma 2 and Theorem 1.

Theorem 2: Consider the asynchronous protocol (3) with structurally fixed topology $F(k)=F, \forall k \in \mathbb{N}$. Assume that all the agents can access their own states (i.e., $F$ has positive diagonal entries) and at least one of the agents can access

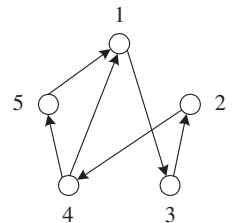

Fig. 1. The interaction topology of an asynchronous multi-agent system.

its own state without delay and the initial value is great than zero. Then the consensus is asymptotically reachable if the associated (directed) graph $G$ has a spanning tree. That is, global consensus is asymptotically reachable under the asynchronous mode.

Remark 4: In the full version of this paper, we show that the irreducibility of $F$ in Lemma 2 can be relaxed in the proof of Theorem 2 using a decomposition idea.

Although Theorems 1 and 2 look similar, they are fundamentally different. In the synchronous case, $x(t)$ converges to a consensus point, which is only a function of the interaction topology and initial states and otherwise independent of the computations; In the latter case, $x(k)$ converges to a consensus point depending on the computations, that is, on the update sets, the delays, and initial states. An intuitive explanation of why this is the case will be given in the next section. The only exception is when the interaction graph has a unique rooted spanning tree, states of all the nodes asymptotically converge to the initial state of the root node.

Example 1: This example shows that the asynchronous consensus value generally depends on the course of the computations. A multi-agent system with five agents and a (structurally fixed and equally weighted) interaction topology is shown in Fig. 1. Since the interaction graph has a directed spanning tree, the consensus is reachable under asynchronous updating. With the initial condition $x(0)=$ $\left[\begin{array}{lllll}-2 & 1 & 2 & 0 & -4\end{array}\right]^{T}$, the asynchronous consensus value of the system is studied. At every iteration, a node is chosen to update its state randomly and independently of other nodes with probability $p$. The delay $d(i, j, k)$ in (3) is a discrete random variable taking an integer value between 0 and $D$ with an equal probability. All randomizations across the nodes and across the iterates are independent in the simulations.

The Monte Carlo simulation experiments are conducted. Fig. 2 shows different consensus values for 2000 independent runs with node selection probability $p=1 / 2$ and the delay bound $D=0$ (zero-asynchronism). From this figure, it is clear that the asynchronous consensus value can take any value in a bounded range.

\section{Synchronous Consensus With TIME-VARYING TOPOLOGIES: An ASYNCHRONOUS PERSPECTIVE}

We have addressed the asynchronous consensus problem under a (structurally) fixed topology, which has the same directed spanning tree during the consensus process. This obviously is a quite restrictive requirement. However, we 


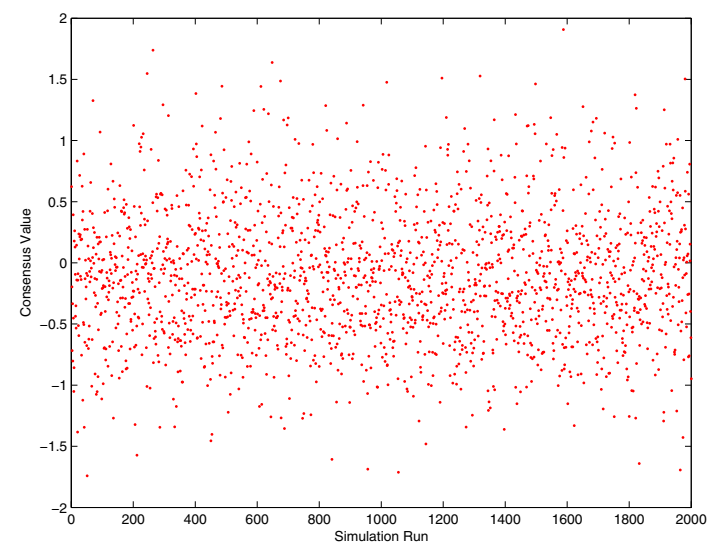

Fig. 2. Asynchronous consensus value $(p=1 / 2$ and $D=0)$ depends on various topology selections made in the course of the computations.

need to emphasize that the topology is only invariant in the "structure domain" or $F(k)=F, \forall k \in \mathbb{N}$ in (3). In the time domain, the interaction between agents may be timedependent due to asynchronisms. Can we map the synchronous systems under time-varying interaction topology into a particular type of asynchronous systems? The answer is affirmative as shown below, thus results from asynchronous systems can be used to study the synchronous consensus protocols. Theorem 2 forms the basis of the following development.

Consider a special type of asynchronous multi-agent systems under a fixed interaction topology. All the agents in the system update their states always at the same time instants (say, when the updating mechanism is triggered by a time impulse coming from an external clock). The asynchronism is solely caused by the loss and creation of communication links between different agents. In fact, this kind of asynchronous systems, with the updating set $S(k)=\{1, \cdots, n\}$ for all $k$ and non-zero delays, can be seen as synchronous systems under time-varying topologies. To see this, we give a simple example. In the sequel, we no longer differentiate the discrete time index $t$ and event based discrete time index $k$.

Example 2: Consider an asynchronous multi-agent system under a (structurally) fixed topology $G_{0}$, which has a directed spanning tree, as shown in $G_{31}$ of Fig. 3(a). The updating sets satisfy $S(t)=\{1,2,3\}$ for all $t$ and time delays satisfy $0 \leq d(i, j, t) \leq D$ with $D$ a finite nonnegative integer. Due to time delays, at a particular time instant the interaction topology may correspond to one of the graphs from Fig. 3(a).

On the other hand, a synchronous multi-agent system under time-varying topologies is shown in Fig. 3(b). At every time instant, the system has an interaction graph which is the superset of one of the graphs from Fig. 3(a), e.g., $G(t+1) \supseteq G_{21}$. Assume that the time interval $\left[t, t+T_{1}\right)$ is long enough such that $\bigcup_{q=0}^{T_{1}} G(t+q)=G_{0}$. Now we see that the synchronous system under time-varying

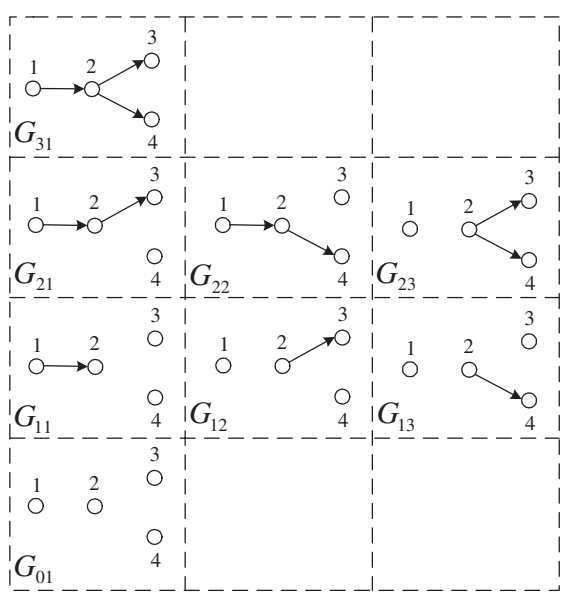

(a)

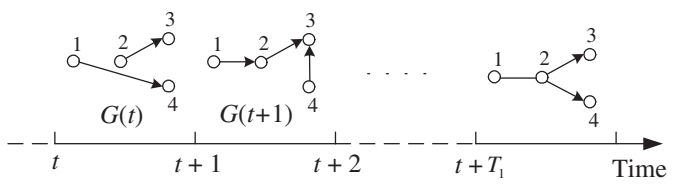

(b)

Fig. 3. Synchronous systems with time varying topologies can be seen as a special case of asynchronous systems.

topologies maps into the asynchronous system across the time interval, thus the two systems have the same stability property in terms of consensus reaching. From the pigeonhole principle, $T_{1}=D C(n)+1$ is enough to guarantee the union of the interaction graphs containing a spanning tree, where $C(n)=2^{n-1}(n=4)$ is the total number of graphs in Fig. 3(a).

In Example 2, the mapping between an asynchronous system and a synchronous system is established by assuming that the union of the synchronous interaction graphs across some time interval contains the same spanning tree as the asynchronous system. This assumption is not restrictive and can be relaxed. For a system with $n$ agents, the total number of different directed spanning tree configurations, denoted as $L(n)$, is finite. Again using the pigeon-hole principle, we can conclude that there must exist the same spanning tree across each time interval of length $T_{2}=(L(n)+1) \times T$, if the union of graphs across each time interval of length $T\left(\leq T_{1}\right)$ has a spanning tree (not necessarily the same one). Note that $T_{2}$ is a finite number since $L(n)$ and $T$ are both bounded. If the asynchronous system under the interaction topology with such a spanning tree can asymptotically reach consensus, so can the synchronous system as $t \rightarrow \infty$.

Based on the above discussions, we have the following result.

Theorem 3: Let $G(t) \in \bar{G}$ be a time-varying interaction graph at time $t$, with the weights selected from a finite set of arbitrary positive numbers. The protocol (3) achieves global consensus asymptotically if and only if there exists an infinite sequence of contiguous, nonempty, bounded time intervals $\left[t_{l}, t_{l+1}\right), l \geq 0$, starting at $t_{0}=0$, with the property 
that across each such interval, the union of the interaction graphs has a spanning tree.

Theorem 3 is in essence the same to Proposition 1 in [26] and Theorem 3.3 in [31] but the proof techniques are totally different. As we see, the same synchronous system (under the time-varying topologies) may be mapped to different asynchronous systems with different spanning trees. This also gives a physical example why the constant $b$ in Lemma 2 is computationally dependent. Tight lower and upper bounds for the constant $b$ are given in [23].

\section{Discussions And Conclusions}

In this paper, we cast existing work on consensus protocols into an asynchronous framework. This extension lends powerful results to the consensus problem. Specifically, we give additional mild conditions in Theorem 2 which when applied to previously known stable consensus protocols add the depth of being provably stable under asynchronous communications.

Some open problems remain. One of them is to understand how asynchronism affects the value of the consensus point (in view of the results of Example 1). For example, it is desirable to control UAVs to visit a pre-specified location within a time window rather than at any time in the planning horizon.

\section{REFERENCES}

[1] T. Arai, E. Pagello, and L. E. Parker, "Guest editorial - Advances in multirobot systems," IEEE Trans. Robot. and Automat., vol. 18, no. 5, pp. 655-661, 2002.

[2] D. Bauso, L. Giarre, and R. Pesenti, "Attitude alignment of a team of UAVs under decentralized information structure," Proc. 2003 IEEE Conf. Contr. Applicat., 2003, pp. 486-491.

[3] R. W. Beard and V. Stepanyan, "Information consensus in distributed multiple vehicle coordinated control," Proc. 42nd IEEE CDC, Maui, HI, 2003, pp. 2029-2034.

[4] G. Beni and P. Liang, "Pattern reconfiguration in swarms - convergence of a distributed asynchronous and bounded iterative algorithm," IEEE Trans. R\&A, vol. 12, no. 3, pp. 485-490, 1996.

[5] D. P. Bertsekas and J. N. Tsitsiklis, Parallel and Distributed Computation: Numerical Methods, Prentice-Hall, Englewood Cliffs, NJ 1989.

[6] G. A. de Castro and F. Paganini, "Convex synthesis of controllers for consensus," Proc. 2004 ACC, Boston, MA, 2004, pp. 4933-4938.

[7] J. Caughman, G. Lafferriere, J. J. P. Veerman, A. Williams, "Decentralized control of vehicle formations," Portland State Preprint, 2004 http://www.mth.pdx.edu/ veerman/flocks1.pdf

[8] D. Chazan and W. Miranker, "Chaotic relaxation," Lin. Algebra and Its Applicat., vol. 2, pp. 199-222, 1969.

[9] J. A. Fax and R. M. Murray, "Information flow and cooperative control of vehicle formations," IEEE Trans. Automat. Contr., vol. 49, no. 9, pp. 1465-1476, 2004.

[10] A. Frommer and D. B. Szyld, "On asynchronous iterations," $J$. Computational and Applied Math., vol. 123, pp. 201-216, 2000.

[11] V. Gazi and K. M. Passino, "Stability of a one-dimensional discretetime asynchronous swarm," Proc. IEEE Int. Symp. Intellig. Contr., Mexico City, Mexico, 2001, pp. 19-24.

[12] Y. Hatano and M. Mesbahi, "Agreement over random networks," Proc. 43rd IEEE CDC, Atlantis, Bahamas, 2004

[13] R. A. Horn and C. R. Johnson, Matrix Analysis, New York: Cambridge University Press, 1985.

[14] A. Jadbabaie, J. Lin, and A. S. Morse, "Coordination of groups of mobile autonomous agents using nearest neighbor rules," IEEE Trans. Automat. Contr., vol 48, no. 6, pp. 988-1001, 2003.

[15] V. S. Kozyakin, "Asynchronous systems: a short survey and problems," http://euclid.ucc.ie/appliedmath/preprints/BCRL 13_03.pdf
[16] N. E. Leonard and E. Fiorelli, "Virtual leaders, artificial potentials and coordinated control of groups," IEEE 40th CDC, Orlando, FL, 2001, pp. 2968-2973

[17] S. Li and H. Wang, "Multi-agent coordination using nearest neighbor rules: Revisiting the Vicsek model," http://arxiv.org/abs/cs/0407021

[18] J. Lin, A. S. Morse, and B. D. O. Anderson, "The multi-agent rendezvous problem - The asynchronous case," Proc. 43rd IEEE CDC, Atlantis, Bahamas, 2004.

[19] Z. Lin, M. Broucke, and B. Francis, "Local control strategies for groups of mobile autonomous agents," IEEE Trans. Automat. Contr., vol. 49, no. 4, pp. 622-629, 2004

[20] Y. Liu, K. Passino, and M. Polycarpou, "Stability analysis of onedimensional asynchronous swarms," Proc. 2001 ACC, Arlington, VA, 2001, pp. 716-721.

[21] — "Stability analysis of one-dimensional asynchronous mobile swarms," Proc. 40th IEEE CDC, Orlando, FL, 2001, pp. 1077-1082.

[22] — "Stability analysis of M-dimensional asynchronous swarms with a fixed communication topology," Proc. 2002 ACC, Anchorage, AK, 2002, pp. 1278-1283

[23] B. Lubachevsky and D. Mitra, "A chaotic asynchronous algorithm for computing the fixed point of a nonnegative matrix of unit spectral radius," J. ACM, vol. 33, no. 1, pp. 130-150, 1986.

[24] N. Lynch, Distributed Algorithms, Morgan Kaufmann, 1996.

[25] M. Mehyar, D. Spanos, J. Pongsajapan, S. Low, and R. Murray, "Distributed averaging on peer-to-peer networks," submitted to IPSN 2005.

[26] L. Moreau, "Leaderless coordination via bidirectional and unidirectional time-dependent communication," Proc. 42nd IEEE CDC, Maui, HI, 2003, pp. 3070-3075.

[27] L. Moreau, "Stability of continuous-time distributed consensus algorithms," Proc. 43rd IEEE CDC, Atlantis, Bahamas, 2004.

[28] R. Olfati-Saber and R. M. Murray, "Consensus problems in networks of agents with switching topology and time-delays," IEEE Trans. Automat. Contr., vol. 49, no. 9, pp. 1520-1533, 2004

[29] A. Pikovsky, M. Rosenblum, and J. Kurths, Synchronization: A Universal Concept in Nonlinear Science, Cambridge University Press, 2003.

[30] M. Pott, "On the convergence of asynchronous iteration methods for nonlinear paracontractions and consistent linear systems," Lin. Algebra and Its Applicat., vol. 283, pp. 1-33, 1998.

[31] W. Ren and R. W. Beard, "Consensus of information under dynamically changing interaction topologies," Proc. 2004 ACC, Boston, MA, 2004, pp. 4939-4944.

[32] W. Ren and R. W. Beard, "Dynamic consensus seeking in distributed multi-agent coordinated control," submitted to IEEE 2004 Int. Conf. $R \& A, 2004$.

[33] A. V. Savkin, "Coordinated collective motion of groups of autonomous mobile robots: Analysis of Vicsek's model," IEEE Trans. Automat. Contr., vol. 49, no. 6, pp. 981-983, 2004.

[34] D. S. Scherber and H. C. Papadopoulos, "Locally constructed algorithms for distributed computations in ad-hoc networks," Proc. ISPN'04, Berkeley, CA, 2004, pp. 11-19.

[35] J. J. E. Slotine and W. Wang, "A study of synchronization and group cooperation using partial contraction theory," Block Island Workshop on Cooperat. Contr., V.J. Kumar, N.E. Leonard, A.S. Morse Eds., Springer-Verlag, 2003.

[36] Y. Su, A. Bhaya, E. Kaszkurewicz, and V. S. Kozyakin, "Further results on convergence of asynchronous linear iterations," Linear Algebra and Applicat., vol. 281, pp. 11-24, 1998.

[37] D. B. Szyld, "The mystery of asynchronous iterations convergence when the spectral radius is one," Report 98-102, Temple University, 1998.

[38] Terrestrial Planet Finder. http://planetquest.jpl.nasa.gov/TPF

[39] F. J. Vazquez-Abad, C. G. Cassandras, and V. Julka, "Centralized and decentralized asynchronous optimization of stochastic discrete-event systems," IEEE Trans. AC, vol. 43, no. 5, pp. 631-655, 1998.

[40] T. Vicsek, A. Czirok, E. Ben-Jacob, I. Cohen, and O. Shochet, "Novel type of phase transition in a system of self-driven particles," Phys. Rev. Lett., vol. 75, no. 6, pp.1226-1229, 1995

[41] L. Xiao and S. Boyd, "Fast linear iterations for distributed averaging," Proc. 42nd IEEE CDC, Maui, HI, 2003, pp. 4997-5002. 